\title{
The Effect of Electroacupuncture on Dynamic Balance during Stair Climbing for Elderly Patients with Knee Osteoarthritis
}

\author{
Meijin Hou $\mathbb{D}^{1,2}$ Xiangbin Wang, ${ }^{2,3}$ Jiao Yu, ${ }^{4}$ Shengxing Fu, ${ }^{2,3}$ Fengjiao Yang, ${ }^{2,3}$ \\ Zhenhui Li, ${ }^{2,3}$ Yanxin Zhang $\mathbb{D}{ }^{2,3,5}$ and Jing Tao $\mathbb{D}^{2,3}$ \\ ${ }^{1}$ National Joint Engineering Research Centre of Rehabilitation Medicine Technology, \\ Fujian University of Traditional Chinese Medicine, Fuzhou, Fujian 350122, China \\ ${ }^{2}$ Key Laboratory of Orthopaedics \& Traumatology of Traditional Chinese Medicine and Rehabilitation (Fujian \\ University of TCM), Ministry of Education, Fuzhou, Fujian 350122, China \\ ${ }^{3}$ College of Rehabilitation Medicine, Fujian University of Traditional Chinese Medicine, Fuzhou, Fujian 350122, China \\ ${ }^{4}$ Department of Rehabilitation Medicine, The First Affiliated Hospital of Fujian Medical University, Longyan, China \\ ${ }^{5}$ Department of Exercise Sciences, Faculty of Science, The University of Auckland, Auckland, New Zealand
}

Correspondence should be addressed to Yanxin Zhang; yanxin.zhang@auckland.ac.nz and Jing Tao; taojing01@fjtcm.edu.cn

Received 3 September 2019; Revised 2 June 2020; Accepted 24 July 2020; Published 13 August 2020

Academic Editor: Adolfo Andrade-Cetto

Copyright (c) 2020 Meijin Hou et al. This is an open access article distributed under the Creative Commons Attribution License, which permits unrestricted use, distribution, and reproduction in any medium, provided the original work is properly cited.

Background. Poor balance is one of the risk factors for falls in patients with knee osteoarthritis (KOA), which is related to the symptoms. Electroacupuncture (EA) is one of the traditional Chinese conservative methods commonly used to improve the symptoms in patients with KOA. Objective. To assess whether EA increases the dynamic balance during stair negotiation among patients with KOA. Methods. A total of $40 \mathrm{KOA}$ patients were assigned to two groups randomly (true electroacupuncture vs. mock electroacupuncture). Acupoints around the knee were selected in the true electroacupuncture (TEA) group with electrical stimulation $(2 \mathrm{~Hz}$ ). In the mock electroacupuncture (MEA) group, about $2 \mathrm{~cm}$ next to the above acupoints, the needles were inserted superficially without electrical stimulation. All the participants received 11 sessions of stimulation treatment in three weeks. The primary outcome was margin of stability (MOS). Secondary outcomes included hip kinematics and kinetics as well as pain. Results. There was no significant difference between the two groups for self-reported pain $(p=0.585)$. During ascent, there was no difference between groups in MOS value in both directions, which was the anterior-posterior $(\mathrm{A} / \mathrm{P})$ direction and medial-lateral $(\mathrm{M} / \mathrm{L})$ direction at initial contact and toe-off as well as the midstance in the gait cycle, and no difference for the hip kinematics and kinetics between the groups was detected $(p>0.05)$. For descent, at the toe-off event, the TEA group was more unstable as compared to the MEA group in the A/P direction $(p=0.029)$ but not in the $\mathrm{M} / \mathrm{L}$ direction, and the hip showed a larger internal rotator moment $(p=0.049)$; at the midstance, the TEA group showed a lower abductor moment than the MEA group $(p=0.003)$. Conclusions. Based on the assessment results from the chosen patients with KOA, the TEA did not demonstrate a significant effect in improving the dynamic balance during stair negotiation in comparison with the MEA. This finding does not support EA as a conservative treatment to improve the dynamic balance in such patients.

\section{Introduction}

Among people age 45 years or older, the overall prevalence of symptomatic knee osteoarthritis (KOA) was $8.1 \%$ [1]. Elderly people suffered a high risk of falls [2], and the fallrelated injuries contributed to a huge economic burden [3]. Elderly patients with KOA were $54 \%$ more likely to experience a fall than those without KOA in the past year [4]. So, the economic burden of elderly KOA patients may be higher than those of their peers.

A recent systematic review shows poor balance is one of the risk factors for falls in KOA patients [5], which is related to symptoms such as pain, muscle weakness, and limitation to range of joint motion [6]. Balance refers to the stability to 
maintain the centre of mass (COM) over the base of support (BOS) [6], and it can be divided into static and dynamic balance. The clinical standing balance tests, such as the Step Test, Single Leg Stance Test, or laboratory-based measurements of standing balance, such as the centre of pressure (COP) velocity and Biodex score, show that KOA patients performed worse with regard to standing balance than healthy controls $[7,8]$. For dynamic balance, it refers to the ability to maintain the COM on the BOS by considering the body position and velocity while doing some dynamic tasks [9], and KOA patients also performed impairments, such as lower Community Balance and Mobility Scale scores as compared to the healthy controls [10]. Conservative treatment, such as transcutaneous electrical nerve stimulation (TENS) simultaneously combined with local heat [11] and electrical stimulation combined with continuous passive motion [12], can relieve pain and potentially improve the dynamic balance in KOA patients during gait. Acupuncture can improve the symptoms and functional activities of KOA patients [13] and alter the gait control strategy toward a normal pattern to meet the demand of supporting the body [14].

But most studies focused on the gait and paid less attention to the effect of the conservative treatment on the dynamic balance during stair negotiation. Stair climbing is a common activity of daily life. The fall risk of stair walking is higher than that of flat walking, especially in stair descent [15]. A previous study used the extrapolated centre of mass (XCOM) method to evaluate the dynamic balance when KOA patients descended stairs and disclosed that older individuals showed reduced dynamic balance control as compared with young individuals [16]. Therefore, the aim of this study was to determine the efficacy of acupuncture combined with electrical stimulation (true electroacupuncture, TEA) and mock electroacupuncture (MEA) for dynamic balance in patients with KOA. We hypothesized that TEA would be better than MEA to improve the dynamic balance in KOA patients during stair climbing, especially in descent.

\section{Methods}

The study is a randomized controlled clinical trial (ChiCTRIIR-16010284) whereby the ethical approval was obtained from the Affiliated Rehabilitation Hospital of Fujian University of Traditional Chinese Medicine Institutional Review Board (No. 2016KY-007-01), and each participant signed an informed consent form voluntarily.

2.1. Participants. Forty patients ( 7 males) aged from 48 to 79 with a diagnosis of bilateral symptomatic KOA, in accordance with the random number table, were assigned into an experimental group (TEA group) and a control group (MEA group).

All participants met the following inclusion criteria: (1) had been diagnosed by a physician with bilateral symptomatic KOA according to the clinical and radiographic criteria proposed by the American College of Rheumatology
[17], (2) had both knees with grade 2 or 3 according to the standards of the Kellgren/Lawrence grade [18] by radiographic examination and(3) maintained the ability to walk and climb stairs without assistance.

Participants were excluded if they met any of the following exclusion criteria: (1) had asymptomatic KOA patients or other neuromusculoskeletal diseases that may affect gait and/or cognitive function, such as lower extremity injury, rheumatoid arthritis, neuropathic arthropathy, stroke, or fracture; (2) accepted intra-articular injection, such as a corticosteroid, in the previous two months; (3) accepted a total knee replacement or planned to have the surgery in the following year; and (4) underwent any biomedical treatment, such as medication, exercise programme or physical therapy, traditional Chinese Medicine therapy, and other rehabilitative therapy in the last week.

2.2. Treatment. Every participant of the experimental group accepted a 30-minute TEA treatment, while the control group received MEA treatment. According to a previous study [19] and the clinical experience of the members of the research group, seven acupoints around the knees, namely Neixiyan (EX-LE 4), Dubi (ST 35), Yanglingquan (GB 34), Yinlingquan (SP 9), Xuehai (SP 10), Liangqiu (ST 34), and Zusanli (ST 36) (Figure 1) were selected and acupunctured with a 1.5-inch single needle $(0.30 \times 40 \mathrm{~mm}$, Wuxi Jiajian Medical Instruments Co., LTD, China) through the skin to a depth of 1.5 inch by an experienced acupuncturist. Then, the needles were connected to electric needling equipment (G6805, Shanghai Medical Instruments Co., LTD, China), of which the $2 \mathrm{~Hz}$ continuous square pulse at a maximal current intensity of patient tolerance was chosen. Each channel of G6805 has two electrodes, and we used two channels. One of the electrodes of the first channel was connected to the needle that was inserted into the Neixiyan, while the other one was connected to Yinlingquan. For the second channel, the electrodes were connected with Yanglingquan and Liangqiu, respectively. During the 30minute TEA stimulation, all the patients reported "de qi" sensations, which is a feeling of heaviness, numbness, or distention at the area of acupoints [20]. In the MEA stimulation, the same needle as the experimental group was pierced into the skin at nearly $0.5 \mathrm{~cm}$ in depth at points that were $2 \mathrm{~cm}$ next to the acupoints and away from the meridian by another experienced acupuncturist. And the electric needling equipment was also connected to the needles with no power. During the 30-minute MEA stimulation, none of the patients had "de qi" sensations.

Both groups were treated for 3 weeks, which consisted of the first week of treatment once a day, continuous treatment of 5 days, and then followed by 2-day rest. The treatment of the second week and the third week were once every other day, three times a week. Finally, every patient underwent a total of 11 sessions of treatment.

2.3. Assessment. Before and after treatment, each participant was assessed for their knee pain using a 5-point Likert-type Western Ontario and McMaster Universities Osteoarthritis Index (WOMAC), where 0 means no pain, 4 means worst 


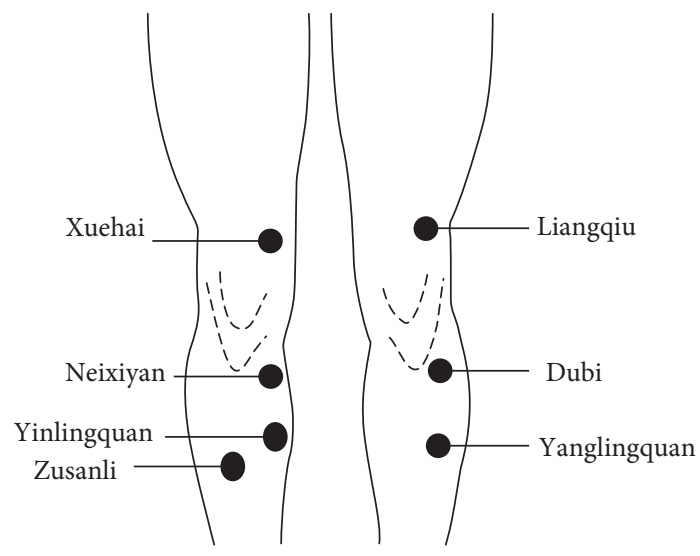

FIgURE 1: The location of acupoints.

imaginable pain, and total score is 20. After the self-reported pain evaluation, all participants were asked to do the stair negotiation test using a three-dimensional gait analysis system (Motion Analysis, Santa Rosa, CA, USA) with a sampling rate of $100 \mathrm{~Hz}$. Passive reflective markers with a diameter of $10 \mathrm{~mm}$ were placed on the skin according to the calibrated anatomical systems technique (CAST) protocol [21]: anterior superior iliac spine, posterior superior iliac spine, great trochanter, medial and lateral femoral epicondyle, medial and lateral malleolus, aspect of the Achilles tendon insertion on the calcaneus, dorsal margin of the first metatarsal head, dorsal aspect of the second metatarsal head, and dorsal margin of the fifth metatarsal head. Two force plates (AMTI BP400600, Advanced Mechanical Technology Inc., Watertown, MA, USA) were used to collect ground reaction force data with a sampling rate of $1000 \mathrm{~Hz}$. The AMTI custom stairs [22] were fixed to the two force plate measure forces on each stair using a standard approach [23]. After familiarization with the test process, each participant performed a stand static trial and then five valid trials of stairs ambulation at a self-selected pace in the step-by-step manner without using the handrails. When participants felt tired, they had a brief rest between trials.

2.4. Data Analysis. Local segment coordinate systems were defined for the pelvis, thigh, shank, and foot segments based on markers' position using Visual 3D Version 6 (C-Motion Inc., Germantown, MA, USA). Joint kinematics were calculated using an $x-y-z$ Cardan angle rotation sequence. Joint kinetics were calculated using inverse dynamics, and the joint moments were normalized to body mass $(\mathrm{Nm} / \mathrm{kg})$. Centre of mass (COM) was calculated using a standard procedure as described by David Winter [24], which was located in the pelvis.

Based on the data of COM displacement, the XCOM values were calculated as follows [25]:

$$
\mathrm{XCOM}=\mathrm{COM}_{\text {position }}+\frac{V_{\mathrm{COM}}}{\sqrt{g / l}},
$$

where $\mathrm{COM}_{\text {position }}$ refers the position of the COM in the anterior-posterior $(\mathrm{A} / \mathrm{P})$ and medial-lateral $(\mathrm{M} / \mathrm{L})$ direction, which was relative to the global coordinate system (GCS) of the LAB, $V_{\mathrm{COM}}$ was estimated by the $\mathrm{A} / \mathrm{P}$ and $\mathrm{M} / \mathrm{L}$ velocity of the COM, $g$ is the gravity $\left(9.8 \mathrm{~m} \cdot \mathrm{s}^{-2}\right)$, and $l$ was the distance between the COP and the COM.

The margin of stability (MOS) value was calculated as follows:

$$
\text { MOS }=\text { BOS }- \text { XCOM }
$$

As the position of the COM within the BOS while postural stability is sustained, a decrease in the MOS indicates that the XCOM exceeds the BOS and stability is therefore disturbed. In other words, larger values indicate greater stability, and negative values represent instability. In our study, we calculated the MOS in the sagittal plane (MOSAP) and the frontal plane (MOSML) using MATLAB 2016a (MathWorks Inc., Natick, MA, USA).

2.5. Statistical Analysis. For all the outcome parameters, namely pain scores, gait velocity and step width and hip joint angles and moments, as well as the MOS in two directions were extracted to do the statistical analysis using the SPSS statistical package Version 20 (SPSS Inc., Chicago, IL, USA), and all significant levels were set at $p<0.05$. The normality of all outcome parameters was initially tested. The analysis of covariance totally has greater statistical power to detect a treatment effect than the other methods [26]. So, we choose the analysis of covariance (ANCOVA) to assess the differences between these two different treatment effects on KOA patients. Each outcome parameters used its own pretreatment data as the covariance.

\section{Results}

36 (6 males) patients finished the research. Four subjects did not finish the study due to a car accident $(n=1)$, scheduling $(n=1)$, measurement rejection $(n=1)$, and removal because of wrong treatment $(n=1)$. After the drop out of 4 subjects from the study, each group had 18 individuals (Figure 2).

There was no significant difference between the groups for age $(61.11 \pm 8.62$ years for the TEA group vs. $65.94 \pm 6.00$ years for the MEA group, $p=0.06)$, BMI $\left(22.89 \pm 2.47 \mathrm{~kg} / \mathrm{m}^{2}\right.$ vs. $\left.24.61 \pm 2.63 \mathrm{~kg} / \mathrm{m}^{2}, \quad p=0.051\right)$, duration of $\mathrm{KOA}$ $(103.42 \pm 113.25$ months for the TEA group vs. $96.67 \pm$ 148.79 months for the MEA group, $p=0.75$ ), and the malefemale ratio $(4 / 14$ vs. $2 / 16, p=0.37)$. For both study and control groups, the pain recorded from posttreatment was significantly improved in comparison with pretreatment. However, no statistically significant differences have been identified (Tables 1 and 2).

3.1. Ascent. The mean MOS value in both $\mathrm{A} / \mathrm{P}$ and $\mathrm{M} / \mathrm{L}$ directions during ascent for each group is shown in Table 1, and there was no between-group difference at initial contact (IC) and toe-off as well as the midstance (MS) in the gait cycle during ascent. After treatment, there was no significant difference between the groups for the velocity $(0.52 \pm 0.06 \mathrm{~m} / \mathrm{s}$ vs. $0.51 \pm 0.07 \mathrm{~m} / \mathrm{s}, \quad p=0.269)$ and step width $(0.08 \pm$ $0.03 \mathrm{~m}$ vs. $0.08 \pm 0.03 \mathrm{~m}, p=0.434)$. Furthermore, no 


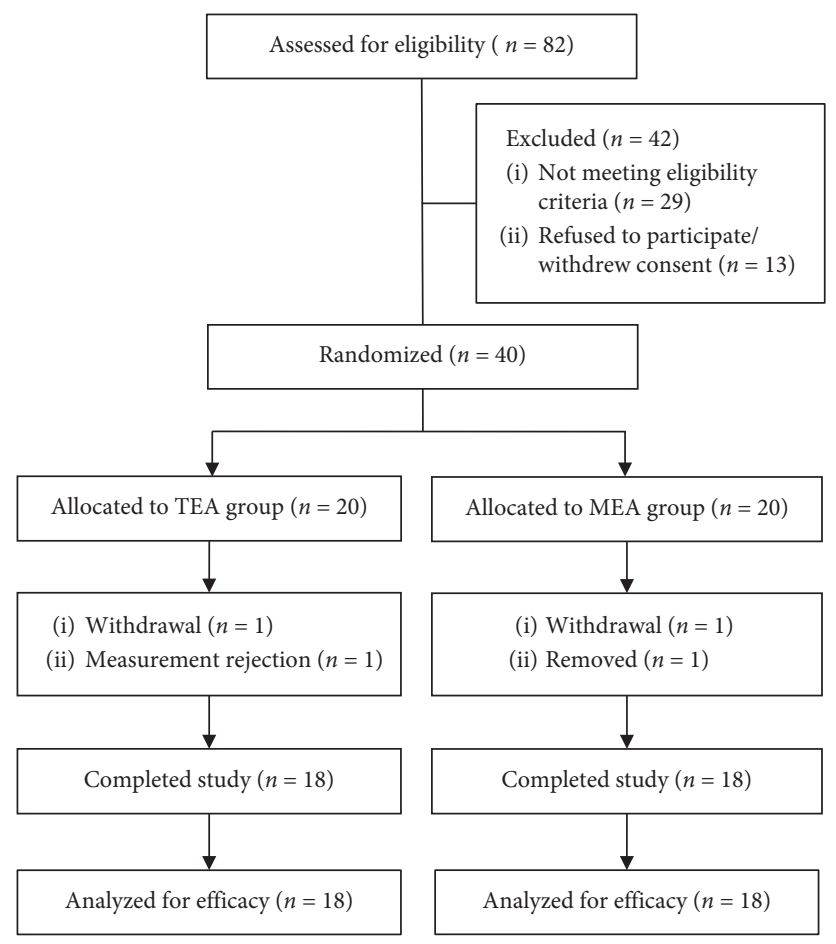

Figure 2: Flow chart of study procedure.

TABLE 1: The pain score and the temporal-distance, kinematic and kinetic variables of the hip during ascent.

\begin{tabular}{|c|c|c|c|c|c|c|c|}
\hline \multirow[t]{2}{*}{ Event } & \multirow[t]{2}{*}{ Variables } & \multicolumn{2}{|c|}{ TEA group (Mean \pm SD) } & \multicolumn{2}{|c|}{ MEA group $($ Mean \pm SD) } & \multicolumn{2}{|c|}{$\begin{array}{l}\text { Comparisons } \\
\text { between groups }\end{array}$} \\
\hline & & Before & After & Before & After & $F$ value & $P$ \\
\hline & Pain & $5.17 \pm 3.68$ & $2.50 \pm 2.20$ & $5.83 \pm 4.36$ & $3.05 \pm 2.60$ & 0.304 & 0.585 \\
\hline & Velocity (m/s) & $0.49 \pm 0.09$ & $0.52 \pm 0.06$ & $0.52 \pm 0.09$ & $0.51 \pm 0.07$ & 1.267 & 0.269 \\
\hline & Step width $(\mathrm{m})$ & $0.09 \pm 0.03$ & $0.08 \pm 0.03$ & $0.08 \pm 0.02$ & $0.08 \pm 0.03$ & 0.626 & 0.434 \\
\hline \multicolumn{8}{|c|}{ Hip angle (degree, $\left.{ }^{\circ}\right)$} \\
\hline & Flex $(+) / \operatorname{Ext}(-)$ & $54.72 \pm 18.95$ & $51.71 \pm 11.49$ & $59.82 \pm 12.56$ & $56.17 \pm 13.06$ & 0.530 & 0.472 \\
\hline \multirow[t]{3}{*}{ IC } & Add $(+) /$ Abd $(-)$ & $7.39 \pm 4.79$ & $6.52 \pm 4.09$ & $6.97 \pm 5.17$ & $6.34 \pm 4.19$ & 0.000 & 0.989 \\
\hline & Int $(+) /$ Ext $(-)$ & $-5.36 \pm 8.69$ & $-2.51 \pm 7.40$ & $-6.89 \pm 8.04$ & $-2.04 \pm 6.58$ & 0.198 & 0.659 \\
\hline & Flex $(+) /$ Ext $(-)$ & $27.05 \pm 10.34$ & $27.16 \pm 14.15$ & $29.54 \pm 9.70$ & $26.92 \pm 8.68$ & 0.256 & 0.616 \\
\hline \multirow[t]{3}{*}{ MS } & Add $(+) / \operatorname{Abd}(-)$ & $5.79 \pm 3.90$ & $3.82 \pm 3.62$ & $3.92 \pm 4.55$ & $3.30 \pm 4.58$ & 0.569 & 0.45677 \\
\hline & Int $(+) / \operatorname{Ext}(-)$ & $-5.11 \pm 9.48$ & $-3.41 \pm 8.46$ & $-5.94 \pm 7.19$ & $-2.21 \pm 6.31$ & 0.631 & 0.433 \\
\hline & Flex $(+) /$ Ext $(-)$ & $20.50 \pm 5.70$ & $16.92 \pm 7.37$ & $19.00 \pm 7.46$ & $20.33 \pm 6.26$ & 2.789 & 0.104 \\
\hline \multirow[t]{2}{*}{ TO } & Add $(+) / \operatorname{Abd}(-)$ & $-1.89 \pm 4.83$ & $-4.84 \pm 3.09$ & $-2.51 \pm 4.50$ & $-3.65 \pm 3.18$ & 2.441 & 0.128 \\
\hline & Int $(+) / \operatorname{Ext}(-)$ & $-6.36 \pm 8.14$ & $-5.61 \pm 7.13$ & $-7.19 \pm 6.78$ & $-4.22 \pm 6.83$ & 0.688 & 0.413 \\
\hline \multicolumn{8}{|c|}{ Hip moment $(\mathrm{Nm} / \mathrm{kg})$} \\
\hline & Flex $(+) / \operatorname{Ext}(-)$ & $-0.0495 \pm 0.1346$ & $-0.0939 \pm 0.1235$ & $-0.0226 \pm 0.1360$ & $-0.0572 \pm 0.1124$ & 0.519 & 0.477 \\
\hline \multirow[t]{3}{*}{ IC } & Add $(+) /$ Abd $(-)$ & $0.0328 \pm 0.0354$ & $0.0236 \pm 0.0398$ & $0.0421 \pm 0.0320$ & $0.0317 \pm 0.0400$ & 0.194 & 0.663 \\
\hline & Int $(+) / \operatorname{Ext}(-)$ & $0.0314 \pm 0.0363$ & $0.0346 \pm 0.0375$ & $0.0444 \pm 0.0351$ & $0.0388 \pm 0.0463$ & 0.015 & 0.903 \\
\hline & Flex $(+) / \operatorname{Ext}(-)$ & $-0.3513 \pm 0.2381$ & $-0.2101 \pm 0.2567$ & $-0.1914 \pm 0.1919$ & $-0.1924 \pm 0.2025$ & 2.076 & 0.159 \\
\hline \multirow[t]{3}{*}{ MS } & Add $(+) /$ Abd $(-)$ & $-0.5913 \pm 0.2111$ & $-0.5727 \pm 0.2023$ & $-0.5267 \pm 0.2006$ & $-0.6107 \pm 0.1357$ & 0.712 & 0.405 \\
\hline & Int $(+) / \operatorname{Ext}(-)$ & $-0.2504 \pm 0.1071$ & $-0.2265 \pm 0.1137$ & $-0.2219 \pm 0.0890$ & $-0.2482 \pm 0.0701$ & 0.643 & 0.429 \\
\hline & Flex $(+) /$ Ext $(-)$ & $-0.0139 \pm 0.1001$ & $-0.0093 \pm 0.0659$ & $0.0603 \pm 0.0756$ & $0.01471 \pm 0.0608$ & 0.117 & 0.734 \\
\hline \multirow[t]{2}{*}{ TO } & Add $(+) /$ Abd $(-)$ & $0.0163 \pm 0.1713$ & $0.0742 \pm 0.0402$ & $0.0617 \pm 0.0562$ & $0.0823 \pm 0.0504$ & 0.232 & 0.633 \\
\hline & Int $(+) / \operatorname{Ext}(-)$ & $-0.0060 \pm 0.0763$ & $0.0115 \pm 0.0256$ & $0.0237 \pm 0.0221$ & $0.0261 \pm 0.0199$ & 2.084 & 0.159 \\
\hline \multicolumn{8}{|c|}{ Margin of stability $(m)$} \\
\hline \multirow{2}{*}{ IC } & $\mathrm{A} / \mathrm{P}$ direction & $0.0926 \pm 0.1251$ & $0.1041 \pm 0.1085$ & $0.0851 \pm 0.1234$ & $0.1123 \pm 0.1073$ & 0.089 & 0.768 \\
\hline & $\mathrm{M} / \mathrm{L}$ direction & $-0.0282 \pm 0.1115$ & $-0.0546 \pm 0.1020$ & $-0.0301 \pm 0.1085$ & $-0.0625 \pm 0.0914$ & 0.057 & 0.813 \\
\hline \multirow{2}{*}{ TO } & $\mathrm{A} / \mathrm{P}$ direction & $-0.1665 \pm 0.0387$ & $-0.1769 \pm 0.0344$ & $-0.1677 \pm 0.0288$ & $-0.1797 \pm 0.0360$ & 0.050 & 0.825 \\
\hline & $\mathrm{M} / \mathrm{L}$ direction & $-0.1344 \pm 0.0281$ & $-0.1390 \pm 0.0290$ & $-0.1334 \pm 0.0291$ & $-0.1380 \pm 0.0317$ & 0.004 & 0.947 \\
\hline \multirow{2}{*}{ MS } & $\mathrm{A} / \mathrm{P}$ direction & $-0.0080 \pm 0.0190$ & $-0.0049 \pm 0.0077$ & $-0.0044 \pm 0.0219$ & $-0.0021 \pm 0.0119$ & 0.610 & 0.440 \\
\hline & $\mathrm{M} / \mathrm{L}$ direction & $-0.0641 \pm 0.0538$ & $-0.0462 \pm 0.0606$ & $-0.0658 \pm 0.0620$ & $-0.0522 \pm 0.0613$ & 0.077 & 0.783 \\
\hline \multirow{2}{*}{$\mathrm{MIN}$} & $\mathrm{A} / \mathrm{P}$ direction & $-0.1642 \pm 0.0396$ & $-0.1741 \pm 0.0363$ & $-0.1637 \pm 0.0343$ & $-0.1767 \pm 0.0394$ & 0.093 & 0.762 \\
\hline & $\mathrm{M} / \mathrm{L}$ direction & $-0.1368 \pm 0.0272$ & $-0.1412 \pm 0.0268$ & $-0.1354 \pm 0.0281$ & $-0.1396 \pm 0.0316$ & 0.012 & 0.914 \\
\hline
\end{tabular}


TABLe 2: The temporal-distance, kinematic, and kinetic variables of the hip during descent.

\begin{tabular}{|c|c|c|c|c|c|c|c|}
\hline \multirow[t]{2}{*}{ Event } & \multirow[t]{2}{*}{ Variables } & \multicolumn{2}{|c|}{ TEA group (Mean \pm SD) } & \multicolumn{2}{|c|}{ MEA group (Mean \pm SD) } & \multicolumn{2}{|c|}{$\begin{array}{l}\text { Comparisons } \\
\text { between groups }\end{array}$} \\
\hline & & Before & After & Before & After & $F$ value & $P$ \\
\hline & Velocity (m/s) & $0.49 \pm 0.09$ & $0.53 \pm 0.05$ & $0.53 \pm 0.10$ & $0.53 \pm 0.08$ & 1.240 & 0.273 \\
\hline & Step width $(\mathrm{m})$ & $0.12 \pm 0.03$ & $0.11 \pm 0.03$ & $0.13 \pm 0.04$ & $0.12 \pm 0.04$ & 0.366 & 0.549 \\
\hline \multicolumn{8}{|c|}{ Hip angle (degree, ${ }^{\circ}$ ) } \\
\hline & Flex $(+) /$ Ext $(-)$ & $25.63 \pm 11.54$ & $20.68 \pm 8.64$ & $27.09 \pm 7.10$ & $24.72 \pm 5.72$ & 2.627 & 0.115 \\
\hline \multirow[t]{3}{*}{ IC } & Add $(+) /$ Abd $(-)$ & $-3.23 \pm 6.26$ & $-4.82 \pm 4.86$ & $-4.50 \pm 5.21$ & $-3.77 \pm 5.05$ & 2.327 & 0.258 \\
\hline & Int $(+) /$ Ext $(-)$ & $-8.95 \pm 9.32$ & $-9.16 \pm 7.14$ & $-10.53 \pm 6.56$ & $-6.72 \pm 6.68$ & 2.491 & 0.124 \\
\hline & Flex $(+) /$ Ext $(-)$ & $20.76 \pm 9.48$ & $17.63 \pm 11.16$ & $26.19 \pm 8.86$ & $22.66 \pm 7.62$ & 0.319 & 0.576 \\
\hline \multirow[t]{3}{*}{ MS } & Add $(+) /$ Abd $(-)$ & $5.02 \pm 3.73$ & $3.25 \pm 2.69$ & $4.30 \pm 4.00$ & $4.89 \pm 3.86$ & 3.123 & 0.086 \\
\hline & Int $(+) /$ Ext $(-)$ & $-4.88 \pm 9.45$ & $-4.144 \pm 9.01$ & $-5.17 \pm 7.18$ & $-1.24 \pm 7.49$ & 1.661 & 0.206 \\
\hline & Flex $(+) /$ Ext $(-)$ & $32.96 \pm 11.56$ & $31.40 \pm 12.26$ & $38.12 \pm 8.42$ & $36.91 \pm 8.00$ & 0.750 & 0.393 \\
\hline \multirow[t]{2}{*}{ TO } & Add $(+) /$ Abd $(-)$ & $1.74 \pm 5.16$ & $0.36 \pm 4.17$ & $2.21 \pm 4.46$ & $1.02 \pm 5.02$ & 0.095 & 0.760 \\
\hline & Int $(+) /$ Ext $(-)$ & $-3.28 \pm 7.22$ & $-2.15 \pm 7.81$ & $-3.21 \pm 7.88$ & $0.62 \pm 6.78$ & 1.658 & 0.207 \\
\hline \multicolumn{8}{|c|}{ Hip moment $(\mathrm{Nm} / \mathrm{kg})$} \\
\hline & Flex (+)/Ext (-) & $0.0546 \pm 0.0855$ & $0.0722 \pm 0.0945$ & $0.0716 \pm 0.0910$ & $0.0909 \pm 0.0750$ & 0.047 & 0.829 \\
\hline \multirow[t]{3}{*}{ IC } & Add $(+) /$ Abd $(-)$ & $0.0043 \pm 0.0420$ & $0.0142 \pm 0.0375$ & $0.0069 \pm 0.0509$ & $0.0112 \pm 0.0487$ & 0.062 & 0.805 \\
\hline & Int $(+) /$ Ext $(-)$ & $0.0085 \pm 0.0182$ & $0.0142 \pm 0.0270$ & $0.0060 \pm 0.0301$ & $0.0090 \pm 0.0224$ & 0.320 & 0.575 \\
\hline & Flex $(+) /$ Ext $(-)$ & $-0.2691 \pm 0.2224$ & $-0.1629 \pm 0.2699$ & $-0.1787 \pm 0.2014$ & $-0.1215 \pm 0.2197$ & 0.238 & 0.629 \\
\hline \multirow[t]{3}{*}{ MS } & Add $(+) / \operatorname{Abd}(-)$ & $-0.7562 \pm 0.1457$ & $-0.6554 \pm 0.1338$ & $-0.7744 \pm 0.2201$ & $-0.7744 \pm 0.0956$ & 10.572 & 0.003 \\
\hline & Int $(+) / \operatorname{Ext}(-)$ & $-0.1777 \pm 0.1128$ & $-0.1296 \pm 0.1313$ & $-0.1915 \pm 0.0807$ & $-0.1675 \pm 0.0807$ & 0.994 & 0.326 \\
\hline & Flex $(+) /$ Ext $(-)$ & $0.1324 \pm 0.1367$ & $0.1473 \pm 0.0962$ & $0.2055 \pm 0.0726$ & $0.2041 \pm 0.0796$ & 2.309 & 0.138 \\
\hline \multirow[t]{2}{*}{ TO } & Add $(+) /$ Abd $(-)$ & $0.0007 \pm 0.0962$ & $0.0184 \pm 0.0882$ & $0.0164 \pm 0.0869$ & $0.0162 \pm 0.0614$ & 0.111 & 0.742 \\
\hline & Int $(+) / \operatorname{Ext}(-)$ & $0.0246 \pm 0.0433$ & $0.0403 \pm 0.0423$ & $0.0020 \pm 0.0358$ & $0.0079 \pm 0.0267$ & 4.192 & 0.049 \\
\hline \multicolumn{8}{|c|}{ Margin of stability $(\mathrm{m})$} \\
\hline \multirow{2}{*}{ IC } & $\mathrm{A} / \mathrm{P}$ direction & $-0.0769 \pm 0.1579$ & $-0.0728 \pm 0.1604$ & $-0.0654 \pm 0.1498$ & $-0.1088 \pm 0.1419$ & 1.009 & 0.323 \\
\hline & $\mathrm{M} / \mathrm{L}$ direction & $-0.0188 \pm 0.1889$ & $-0.0483 \pm 0.2012$ & $-0.0728 \pm 0.2172$ & $-0.0255 \pm 0.2118$ & 0.687 & 0.413 \\
\hline \multirow{2}{*}{ TO } & $\mathrm{A} / \mathrm{P}$ direction & $-0.2328 \pm 0.0228$ & $-0.2451 \pm 0.0264$ & $-0.2196 \pm 0.0681$ & $-0.2107 \pm 0.0544$ & 5.199 & 0.029 \\
\hline & $\mathrm{M} / \mathrm{L}$ direction & $-0.2375 \pm 0.0299$ & $-0.2470 \pm 0.0333$ & $-0.2212 \pm 0.0873$ & $-0.2269 \pm 0.0756$ & 1.221 & 0.277 \\
\hline \multirow{2}{*}{ MS } & $\mathrm{A} / \mathrm{P}$ direction & $-0.0186 \pm 0.0281$ & $-0.0270 \pm 0.0365$ & $-0.0255 \pm 0.0351$ & $-0.0243 \pm 0.0365$ & 0.292 & 0.593 \\
\hline & $\mathrm{M} / \mathrm{L}$ direction & $-0.0687 \pm 0.0895$ & $-0.0881 \pm 0.1024$ & $-0.0858 \pm 0.1010$ & $-0.0656 \pm 0.0956$ & 1.259 & 0.270 \\
\hline \multirow{2}{*}{ MIN } & $\mathrm{A} / \mathrm{P}$ direction & $-0.2493 \pm 0.0271$ & $-0.2597 \pm 0.0322$ & $-0.2537 \pm 0.0350$ & $-0.2519 \pm 0.0259$ & 1.456 & 0.236 \\
\hline & $\mathrm{M} / \mathrm{L}$ direction & $-0.2639 \pm 0.0253$ & $-0.2765 \pm 0.0237$ & $-0.2347 \pm 0.1242$ & $-0.2726 \pm 0.0262$ & 0.317 & 0.577 \\
\hline
\end{tabular}

difference for the hip kinematics and kinetics between the groups was detected $(p>0.05)$ (Table 1$)$.

3.2. Descent. After treatment, the velocity of stair descent showed no significant difference between the two groups $(0.53 \pm 0.05 \mathrm{~m} / \mathrm{s}$ vs. $0.53 \pm 0.08 \mathrm{~m} / \mathrm{s}, p=0.273)$, with the TEA group walking faster than before. The same situation was shown in the step width $(0.11 \pm 0.03 \mathrm{~m}$ vs. $0.12 \pm 0.04 \mathrm{~m}$, $p=0.549$ ). Table 2 also shows the details of the MOS value in both directions and the hip variables. At the initial contact, the MOS, as well as the hip kinematics and kinetics, did not significantly differ between the TEA group and MEA group $(p>0.05)$. At the toe-off, the TEA group was more unstable than the MEA group in the $\mathrm{A} / \mathrm{P}$ direction during stair descent $(-0.2451 \pm 0.0264 \mathrm{~m}$ vs. $-0.2107 \pm 0.0544 \mathrm{~m}$, $p=0.029$ ) but did not differ in the $\mathrm{M} / \mathrm{L}$ direction, and the hip showed a larger internal rotator moment in TEA group after treatment comparing with the MEA group (0.0403 \pm $0.0423 \mathrm{Nm} / \mathrm{kg}$ vs. $0.0079 \pm 0.0267 \mathrm{Nm} / \mathrm{kg}, p=0.049$ ). At the midstance, the TEA group showed a lower abductor moment than the MEA group $(-0.6554 \pm 0.1338 \mathrm{Nm} / \mathrm{kg}$ vs. $-0.7744 \pm 0.0956 \mathrm{Nm} / \mathrm{kg}, p=0.003)$.

\section{Discussion}

The purpose of our study was to investigate the efficacy of TEA for dynamic balance during stair walking in patients with KOA. We found that the effect of TEA was limited for dynamic balance in KOA patients during descent stair walking, and no more efficacious than MEA during ascent stair walking.

For KOA patients, poor balance is related to pain and muscle weakness [6]. A network meta-analysis showed that acupuncture with electrical stimulation or heat pain could relieve symptoms in most KOA patients and may be better than other acupuncture methods [27]. The main effect of EA on KOA was to ease the pain and remarkably improve physical function [28]. However, there was no significant difference in pain scores between the TEA group and MEA group in our study, and it did not agree with those reviews and two studies with similar interventions $[29,30]$. One possible reason for the discrepancy is the MEA setting in acupuncture trials, which is still controversial because the nonpenetrating and superficial acupuncture needle techniques are not totally inert. Thus, MEA may have placebo effects [31]. Furthermore, acupuncture has a convergence 
effect, which means that its effect may be weakened with the prolongation of intervention time, and the effect will be different if the acupoints are selected differently. Plaster et al. [32] studied patients with KOA and indicated that both electroacupuncture and manual acupuncture cannot increase muscle strength. It may be the reason why TEA cannot improve the dynamic balance of patients with KOA. The main strategy used to control the gait balance is the hip strategy, as the hips play a significant role in stabilizing the $\mathrm{COM}$ both in the $\mathrm{A} / \mathrm{P}$ and $\mathrm{M} / \mathrm{L}$ directions [33]. Metaanalysis revealed that KOA patients may adopt kinematic and kinetic alterations during stair climbing, such as increasing hip/trunk flexion angle [34]. Early-stage KOA patients with knee pain may decrease their hip abductor muscle strength when negotiating stairs [35]. The results showed that there were little remarkable differences in hip angle and moments between the two groups. Exercise programmes that focused on lower limb muscle strength training were beneficial for patients with KOA to improve dynamic balance using a modified Star Excursion Balance Test [36]. Therefore, the combination of electroacupuncture and hip muscle strength training may be more effective for KOA patients to improve their dynamic balance.

Most studies $[7,8,10]$ assess dynamic balance by using scales or balance tests, and fewer studies focused on dynamic balance when individuals do some daily activities, such as stair climbing. The demands for dynamic balance control during negotiation of stairs are higher than level walking. A previous study used the XCOM method to evaluate the dynamic balance for descent stair walking [16]. The dynamic stability index shows that speed is an important factor for dynamic stability assessment [37]. Slower walking velocities increase the stability [38]. Gait speed shows a strong correlative relationship with COM velocity [39]. The results of this study showed that the speed of the TEA group during ascent and descent increased after treatment. Although the difference between TEA and MEA was not statistically significant, the possible influence of speed on dynamic stability should still be considered. Due to the speed increase after treatment, the XCOM should be greater and the MOS should be less. This is in agreement with our ascent stair walking results. Even though the speed increased after treatment, there was no significant difference in the MOS values. But for descent stair walking, the MOS in the $\mathrm{A} / \mathrm{P}$ direction at the toe-off event after treatment was more unstable than before its treatment. This may be related to the greater balance control needs in descent as compared with ascent [40].

This study had several limitations. One of the limitations was that all participants accepted the fixed TEA or MEA treatment. Subject-specified acupuncture treatment might be more effective for patients with KOA. Treatment according to syndrome differentiation plays an important part in traditional Chinese medicine. Acupuncture acupoints need to be selected according to the syndrome differentiation. However, it was prohibited in our study. The second limitation was that we did not assess other balancerelated factors, such as muscle strength.

The third limitation was related to the use of MEA. Most of the Chinese people are familiar with acupuncture and understand the feeling of acupuncture, so it is difficult to do
MEA. The position of acupoints and "de qi" sensations are the key points for acupuncture's effect. We need to deviate from the position of acupoints as much as possible and reduce the subjects' sensations to the greatest extent. Nevertheless, the multiple factors in the MEA group (depth and position of acupuncture and the electrical stimulation intensity) may have some treatment effect. In future research, these factors need to be better controlled for the MEA group.

\section{Conclusion}

Based on the assessment results from the chosen patients with KOA, the TEA did not demonstrate a significant effect in improving the dynamic balance during stair negotiation in comparison with the MEA. This finding does not support electroacupuncture as a conservative treatment to improve the dynamic balance in such patients.

\section{Data Availability}

The data that support the findings of this study are available on request from the first author, Meijin Hou.

\section{Conflicts of Interest}

The authors declare no conflicts of interest.

\section{Authors' Contributions}

XBW and JT conceived and designed the study. MJH performed the experiments, analysed the data and results, and wrote the paper. JY assisted in recruiting participants and collecting motion data. SXF, FJY, and ZHL assisted with data extraction. YXZ developed the MATLAB code and reviewed the paper. All authors read and approved the final manuscript.

\section{Acknowledgments}

The authors would like to express appreciation to Anmin Liu and Yunru Ma for reviewing this paper. This research was funded by the National Natural Science Foundation of China (Grant no. 81273819), Science and Technology Platform Construction Project of the Fujian Science and Technology Department (Grant no. 2015Y2001), and the Fujian Key Laboratory of Rehabilitation Technology.

\section{References}

[1] X. Tang, S. Wang, S. Zhan et al., "The prevalence of symptomatic knee osteoarthritis in China: results from the China Health and Retirement Longitudinal Study," Arthritis \& Rheumatology, vol. 68, no. 3, pp. 648-653, 2016.

[2] S. G. Qi, Z. H. Wang, L. M. Wang, and M. Zhang, "Current status of falls and related injuries among Chinese elderly in 2013," Zhonghua Liu Xing Bing Xue Za Zhi, vol. 39, no. 4, pp. 439-442, 2018.

[3] K. Peng, M. Tian, M. Andersen et al., "Incidence, risk factors and economic burden of fall-related injuries in older Chinese people: a systematic review," Injury Prevention, vol. 25, no. 1, pp. 4-12, 2019. 
[4] T. O. Smith, E. Higson, M. Pearson, and M. Mansfield, "Is there an increased risk of falls and fractures in people with early diagnosed hip and knee osteoarthritis? Data from the osteoarthritis initiative," International Journal of Rheumatic Diseases, vol. 21, no. 6, pp. 1193-1201, 2016.

[5] D. G. Manlapaz, G. Sole, P. Jayakaran, and C. M. Chapple, "Risk factors for falls in adults with knee osteoarthritis: a systematic review," PMぬR, vol. 11, no. 7, pp. 745-757, 2019.

[6] K. Bennell, R. S. Hinman, T. V. Wrigley, M. W. Creaby, and P. Hodges, "Exercise and osteoarthritis: cause and effects," Comprehensive Physiology, vol. 1, no. 4, pp. 1943-2008, 2011.

[7] G. L. Hatfield, M. Adam, M. Wenman, C. A. Hammond, and M. A. Hung, "Clinical tests of standing balance in the knee osteoarthritis population: systematic review and meta-analysis," Physical Therapy, vol. 96, no. 3, pp. 324-337, 2015.

[8] T. Lawson, A. Morrison, S. Blaxland, M. Wenman, C. G. Schmidt, and M. A. Hunt, "Laboratory-based measurement of standing balance in individuals with knee osteoarthritis: a systematic review," Clinical Biomechanics, vol. 30, no. 4, pp. 330-342, 2015.

[9] Y.-C. Pai and J. Patton, "Center of mass velocity-position predictions for balance control," Journal of Biomechanics, vol. 30, no. 4, pp. 347-354, 1997.

[10] J. Takacs, S. J. Garland, M. G. Carpenter, and M. A. Hunt, "Validity and reliability of the community balance and mobility scale in individuals with knee osteoarthritis," Physical Therapy, vol. 94, no. 6, pp. 866-874, 2014.

[11] T. Maeda, H. Yoshida, T. Sasaki, and A. Oda, "Does transcutaneous electrical nerve stimulation (TENS) simultaneously combined with local heat and cold applications enhance pain relief compared with TENS alone in patients with knee osteoarthritis?," Journal of Physical Therapy Science, vol. 29, no. 10, pp. 1860-1864, 2017.

[12] F. Tok, K. Aydemir, F. Peker, İ. Safaz, M. A. Taşkaynatan, and A. Özgül, "The effects of electrical stimulation combined with continuous passive motion versus isometric exercise on symptoms, functional capacity, quality of life and balance in knee osteoarthritis: randomized clinical trial," Rheumatology International, vol. 31, no. 2, pp. 177-181, 2011.

[13] X. Lin, K. Huang, G. Zhu, Z. Huang, A. Qin, and S. Fan, “The effects of acupuncture on chronic knee pain due to osteoarthritis," The Journal of Bone and Joint Surgery, vol. 98, no. 18, pp. 1578-1585, 2016.

[14] Y.-H. Liu, I.-P. Wei, T.-M. Wang, T.-W. Lu, and J.-G. Lin, "Immediate effects of acupuncture treatment on intra- and inter-limb contributions to body support during gait in patients with bilateral medial knee osteoarthritis," The American Journal of Chinese Medicine, vol. 45, no. 1, pp. 23-35, 2017.

[15] K. Wang, K. Delbaere, M. A. D. Brodie et al., "Differences between gait on stairs and flat surfaces in relation to fall risk and future falls," IEEE Journal of Biomedical and Health Informatics, vol. 21, no. 6, pp. 1479-1486, 2017.

[16] Y. Koyama, H. Tateuchi, R. Nishimura et al., "Relationships between performance and kinematic/kinetic variables of stair descent in patients with medial knee osteoarthritis: an evaluation of dynamic stability using an extrapolated center of mass," Clinical Biomechanics, vol. 30, no. 10, pp. 1066-1070, 2015.

[17] R. Altman, E. Asch, D. Bloch et al., "Development of criteria for the classification and reporting of osteoarthritis: classification of osteoarthritis of the knee," Arthritis \& Rheumatism, vol. 29, no. 8, pp. 1039-1049, 1986.
[18] J. H. Kellgren and J. S. Lawrence, "Radiological assessment of osteo-arthrosis," Annals of the Rheumatic Diseases, vol. 16, no. 4, pp. 494-502, 1957.

[19] T. W. Lu, I. P. Wei, Y. H. Liu et al., "Immediate effects of acupuncture on gait patterns in patients with knee osteoarthritis," Chinese Medical Journal, vol. 123, no. 2, pp. 165-172, 2010.

[20] P. White, P. Prescott, and G. Lewith, "Does needling sensation (de qi) affect treatment outcome in pain? Analysis of data from a larger single-blind, randomised controlled trial," Acupuncture in Medicine, vol. 28, no. 3, pp. 120-125, 2010.

[21] A. Cappozzo, F. Catani, U. Della Croce, and A. Leardini, "Position and orientation in space of bones during movement: anatomical frame definition and determination," Clinical Biomechanics, vol. 10, no. 4, pp. 171-178, 1995.

[22] U. D. Croce and P. Bonato, "A novel design for an instrumented stairway," Journal of Biomechanics, vol. 40, no. 3, pp. 702-704, 2007.

[23] A. Z. Alshawabka, A. Liu, S. F. Tyson, and R. K. Jones, "The use of a lateral wedge insole to reduce knee loading when ascending and descending stairs in medial knee osteoarthritis patients," Clinical Biomechanics, vol. 29, no. 6, pp. 650-656, 2014.

[24] D. A. Winter, Biomechanics and Motor Control of Human Movement, University of Waterloo Press, Waterloo, Canada, Fourth edition, 1987.

[25] A. L. Hof, M. G. J. Gazendam, W. E. Gazendam, and W. E. Sinke, "The condition for dynamic stability," Journal of Biomechanics, vol. 38, no. 1, pp. 1-8, 2005.

[26] A. J. Vickers and D. G. Altman, "Statistics notes: analysing controlled trials with baseline and follow up measurements," BMJ, vol. 323, no. 7321, pp. 1123-1124, 2001.

[27] S. Li, P. Xie, Z. Liang et al., "Efficacy comparison of five different acupuncture methods on pain, stiffness, and function in osteoarthritis of the knee: a network meta-analysis," Evidence-Based Complementary and Alternative Medicine, vol. 2018, Article ID 1638904, 19 pages, 2018.

[28] N. Chen, J. Wang, A. Mucelli, X. Zhang, and C. Wang, "Electro-acupuncture is beneficial for knee osteoarthritis: the evidence from meta-analysis of randomized controlled trials," The American Journal of Chinese Medicine, vol. 45, no. 5, pp. 965-985, 2017.

[29] S. Ahsin, S. Saleem, A. M. Bhatti, R. K. Iles, and M. Aslam, "Clinical and endocrinological changes after electro-acupuncture treatment in patients with osteoarthritis of the knee," Pain, vol. 147, no. 1, pp. 60-66, 2009.

[30] T. W. Lu, I. P. Wei, Y. H. Liu et al., "Immediate effects of acupuncture on gait patterns in patients with knee osteoarthritis," Chinese Medical Journal, vol. 123, no. 2, pp. 165-172, 2010.

[31] X.-K. Wu, E. Stener-Victorin, H.-Y. Kuang et al., "Effect of acupuncture and clomiphene in Chinese women with polycystic ovary syndrome," JAMA, vol. 317, no. 24, pp. 25022514, 2017.

[32] R. Plaster, W. B. Vieira, F. A. D. Alencar, E. Y. Nakano, and R. E. Liebano, "Immediate effects of electroacupuncture and manual acupuncture on pain, mobility and muscle strength in patients with knee osteoarthritis: a randomised controlled trial," Acupuncture in Medicine, vol. 32, no. 3, pp. 236-241, 2014.

[33] D. Winter, "Human balance and posture control during standing and walking," Gait \& Posture, vol. 3, no. 4, pp. 193-214, 1995. 
[34] H. Iijima, K. Shimoura, T. Aoyama, and M. Takahashi, "Biomechanical characteristics of stair ambulation in patients with knee OA: a systematic review with meta-analysis toward a better definition of clinical hallmarks," Gait \& Posture, vol. 62, pp. 191-201, 2018.

[35] Y. Suzuki, H. Iijima, K. Shimoura, T. Tsuboyama, and T. Aoyama, "Patients with early-stage knee osteoarthritis and knee pain have decreased hip abductor muscle strength while descending stairs," Clinical Rheumatology, vol. 38, no. 8, pp. 2249-2254, 2019.

[36] L. Al-Khlaifat, L. C. Herrington, S. F. Tyson, A. Hammond, and R. K. Jones, "The effectiveness of an exercise programme on dynamic balance in patients with medial knee osteoarthritis: a pilot study," The Knee, vol. 23, no. 5, pp. 849-856, 2016.

[37] T. Caderby, E. Yiou, N. Peyrot, M. Begon, and G. Dalleau, "Influence of gait speed on the control of mediolateral dynamic stability during gait initiation," Journal of Biomechanics, vol. 47, no. 2, pp. 417-423, 2014.

[38] S. A. England and K. P. Granata, "The influence of gait speed on local dynamic stability of walking," Gait \& Posture, vol. 25, no. 2, pp. 172-178, 2007.

[39] A. Lee, T. Bhatt, R. Smith-Ray, E. Wang, and Yi-C. Pai, "Gait speed and dynamic stability decline accelerates only in late life," Journal of Geriatric Physical Therapy, vol. 42, no. 2, pp. 73-80, 2018.

[40] B. J. McFadyen and D. A. Winter, "An integrated biomechanical analysis of normal stair ascent and descent," Journal of Biomechanics, vol. 21, no. 9, pp. 733-744, 1988. 